\title{
The Effect of Perampanel on Resting-State Electroencephalography Functional Connectivity in Patients with Epilepsy
}

\author{
Soo-Hyung Lee ${ }^{1}$, Seon-Jae Ahn, MD, MS ${ }^{2}$, Kwang Su Cha, $\mathrm{MS}^{3}$, Sang Kun Lee, MD, PhD ${ }^{1,3}$, \\ Ki-Young Jung, $\mathrm{MD}, \mathrm{PhD}^{1,3,4}$ \\ ${ }^{1}$ Seoul National University College of Medicine, Seoul, Korea \\ ${ }^{2}$ Center for Hospital Medicine, Seoul National University Hospital, Seoul, Korea \\ ${ }^{3}$ Department of Neurology, Seoul National University Hospital, Seoul, Korea \\ ${ }^{4}$ Neuroscience Research Institute, Seoul National University College of Medicine, Seoul, Korea
}

Received: February 23, 2021

Revised: February 24, 2021

Accepted: March 20, 2021

Corresponding author:

Ki-Young Jung, $\mathrm{MD}, \mathrm{PhD}$

Department of Neurology, Seoul National

University Hospital, Neuroscience

Research Institute, Seoul National

University College of Medicine, 101

Daehak-ro, Jongno-gu, Seoul 03080,

Korea

Tel: $+82-2-2072-4988$

Fax: + 82-2-2072-2474

E-mail: jungky@snu.ac.kr
Background: The purpose of this study was to investigate the changes in electroencephalography (EEG) functional connectivity (FC) induced by perampanel treatment and to explore the relationship between changes in FC and serum perampanel levels.

Methods: The EEG recordings of 16 patients with epilepsy were used in this study. EEG recordings and neuropsychological tests were performed before and after 25 weeks of perampanel treatment. Serum perampanel levels were also obtained after treatment. FC was measured using the weighted phase lag index (wPLI) and was compared before and after treatment. The correlation between significant changes FC and serum perampanel levels was evaluated.

Results: Eight patients showed a more than 50\% reduction in seizure frequency. However, neuropsychological tests showed little change, with only the delayed recall of the Rey Complex Figure showing a significant increase after perampanel treatment. In FC analysis, the wPLI of three electrode pairs (FP1-P3, F8-P3, and P4-Pz) in the alpha band significantly decreased. The changes in the connectivity of the electrode pairs were not correlated with serum perampanel levels.

Conclusions: Although decreases in cognitive function were not observed due to perampanel treatment, significant reductions were observed in alpha band FC, suggesting a potential negative effect on cognition.

Keywords: Electroencephalography; Epilepsy; Anticonvulsants; Perampanel; Functional connectivity

\section{Introduction}

Perampanel is an antiepileptic drug (AED) usually used as primary and adjunctive treatment for partial onset seizures with or without secondary generalized tonic-clonic seizures and as adjunctive treatment in generalized tonic-clonic seizures. Perampanel acts selectively on the $\alpha$-amino-3-hydroxy-5-methyl-4-isoxazolepropionic acid receptor (AMPAR) as a noncompetitive an- tagonist. ${ }^{1,2}$ Because AMPAR is an ionotropic transmembrane receptor for glutamate-mediated excitatory neurotransmission in the central nervous system, AMPAR inhibition caused by perampanel may negatively affect cognitive functions.

A study comprising subjects aged 12 to 18 years reported that the continuity of attention and speed of memory decreased and the quality of episodic memory increased after 19 weeks of adjunctive perampanel treatment, although the effect size was

This is an Open Access article distributed under the terms of the Creative Commons Attribution Non-Commercial License (http://creativecommons.org/licenses/by-nc/4.0/) which permits unrestricted non-commercial use, distribution, and reproduction in any medium, provided the original work is properly cited.

Copyright (C) 2021 Korean Clinical Epilepsy Research Society 
small. ${ }^{3}$ After 52 weeks using the same subjects, no change in the speed of memory was observed, while a small but statistically significant decrease in the power of attention was found, suggesting that perampanel may have negatively affect attention. ${ }^{4} \mathrm{An}$ other study reported small increases in the power of attention and quality of episodic secondary memory, together with decreases in the continuity of attention related to increased perampanel serum levels in adolescents aged 12 to 18 years. ${ }^{5}$ Two studies reported no cognitive deficits due to perampanel treatment. ${ }^{6,7}$ In our previous study comprising adult patients, although neuropsychological (NP) tests showed no decline in cognition, electroencephalography (EEG) spectral analysis showed an increase in the power of the theta band and a decrease in the alpha band, accompanied by a decrease in the peak alpha frequency after 6 months of perampanel treatment, indicating potential negative effects on cognitive function. ${ }^{7}$ However, another study using EEG spectral analysis reported an increase in cortical EEG fast activity, with an increase in the beta band and a decrease in the delta band in adults and children after three months of follow-up, indicating potential enhancement of attention and cognition. ${ }^{8}$ Although previous studies using comprehensive NP tests, a gold standard for examining cognitive function, have reported little or no change in cognition after perampanel treatment, significant changes in the power of brain oscillations were observed. Considering that brain oscillations are more sensitive than clinical measures, further investigation is needed to evaluate the effect of perampanel on brain activity.

Neuronal coherence is recognized as a sign of interaction or communication between active neuronal groups; thus, it is closely related to cognition. ${ }^{9}$ Functional connectivity (FC) is the temporal correlation of activity between different brain regions, quantitatively representing neuronal coherence. Naturally, FC measures can be used to investigate alterations in cognitive function. ${ }^{10}$ Little is known thus far about the effect of perampanel on $\mathrm{FC}$, and knowledge concerning the FC change induced by perampanel may lead to a better understanding of the effect of perampanel on brain activity and cognition.

In the present study, we analyzed phase-based FC in EEG caused by perampanel signals. We sought to scrutinize the changes in EEG FC and determine the relationship between the changes in FC and perampanel serum levels. The weighted phase lag index (wPLI) was used as a measure of FC to minimize the effect of volume conduction and reduce spurious connections.

\section{Methods}

\section{Patients}

This study was approved by the Institutional Review Board of Seoul National University Hospital (No. C-1602-107-742), and informed consent was obtained from all the participants.

The patient characteristics and study design were described in our previous study. The patients were aged between 19 and 65 years and had partial seizures with or without secondary generalized seizures. The EEGs were recorded, and NP tests were conducted for all the participants before they started taking perampanel (baseline) and after 25 weeks (follow-up). Perampanel was taken orally once a day, and the dose was increased by at least 2 $\mathrm{mg}$ every 2 weeks, starting at $2 \mathrm{mg} /$ day. The doses were increased by $2 \mathrm{mg}$ every week for patients taking enzyme-inducing antiepileptic drugs concurrently. The maintenance dose of perampanel ranged from 4 to $12 \mathrm{mg} /$ day, which was not changed for a month before the final EEG recording. Concomitant AEDs were not changed during study period.

\section{Electroencephalography recording and processing}

The EEGs were recorded using the International 10-20 system with cap electrodes (Natus Neurology Inc., Middleton, WI, USA). The impedance of all the electrodes was kept below $10 \mathrm{k} \Omega$. Resting-state EEGs were recorded for 10 minutes at a sampling rate of $400 \mathrm{~Hz}$ while patients were seated comfortably. Patients were asked to close their eyes and open them every 10 seconds. For each EEG recording with the eyes closed, 3-second-long epochs were selected when the posterior dominant rhythm was present in the occipital regions. An average of 33. ${ }^{1}$ epochs was selected from each EEG recording.

EEG analyses were performed using MATLAB 2019b (MathWorks Inc., Natick, MA, USA). The EEG data were re-referenced to the common average reference and bandpass filtered $(0.5-50 \mathrm{~Hz})$ for analysis. Direct current (DC) offset removal and detrending were applied to eliminate DC fluctuations and deterministic trends. Independent component analysis was applied to correct stereotyped ocular and muscular artifacts. The preprocessed EEG data from a previous study were used in this study.

\section{Functional connectivity analysis}

The wPLI was used as a measure of FC. The wPLI is a phasebased measure of FC similar to the phase lag index (PLI). The PLI measures the asymmetry of the distribution of phase differences between two electrodes. ${ }^{11}$ Based on spurious connectivity due to volume conduction likely having a phase angle difference 
of 0 or $\pi$, PLI is defined as the extent to which the distribution of phase angle differences is asymmetric. It is calculated by taking the average of the sign of the imaginary part of the cross-spectrum. Although true connections with a phase angle difference close to 0 or $\pi$ may not be found when the PLI is used, it effectively minimizes the effect of volume conduction; thus, it is an effective means for assessing FC in exploratory research. The wPLI gives weights to phase angle differences that are farther away from 0 or $\pi$ by weighting the sign of the phase angle differences by the magnitude of the imaginary component, further reducing spurious connections.

In this study, the EEG recordings were first bandpass filtered to obtain four frequency bands; delta $(0.5-4.0 \mathrm{~Hz})$, theta $(4-8$ $\mathrm{Hz})$, alpha $(8-12 \mathrm{~Hz})$, and beta $(12-30 \mathrm{~Hz})$. A Hilbert transform was applied to each epoch in each of the four frequency bands in every EEG recording to obtain the instantaneous phase for each time point. Next, the wPLI was calculated for each electrode pair. The wPLI matrices for each patient at baseline and follow-up were obtained by averaging the wPLI over all epochs.

\section{Neuropsychological examinations}

The NP test scores from a previous study were used in the present study. ${ }^{7}$ The Beck Depression Inventory-II was used to assess the degree of depression. ${ }^{12}$ The Epworth Sleepiness Scale was used to measure daytime sleepiness, ${ }^{13}$ and the Buss-Durkee Hostility Inventory was used to measure aggression. ${ }^{14}$ To evaluate general cognitive function, the Mini-Mental Status Examination was used. ${ }^{15}$ For language function measurement, the Korean Boston Naming Test was used. ${ }^{16}$ The Rey Complex Figure Test (RCFT) was administered to evaluate visuospatial and memory function, using the copy score and copy time for visuospatial measurement and immediate recall, delayed recall, and recognition for memory function evaluation. ${ }^{17}$ The Korean California Verbal Learning Test was used to assess verbal and working memory functions. ${ }^{18}$ To measure attention and executive functions, the digit span forward and backward tests, Controlled Oral Word Association Test, ${ }^{19}$ and Trail Making Test were used. ${ }^{20}$

\section{Perampanel serum level analysis}

The perampanel serum level data were obtained from a previous study. ${ }^{7}$ High-performance liquid chromatography (HPLC; 1200 series, Agilent Technologies, Santa Clara, CA, USA) coupled with tandem mass spectrometry (MS/MS; API3200, Applied Biosystems, Waltham, MA, USA) was used to measure the perampanel serum levels. Fifty microliters of plasma was mixed with $50 \mu \mathrm{L}$ of an internal standard and $100 \mathrm{ng} / \mathrm{mL}$ of trazodone in $50 \%$ methanol. Next, $300 \mu \mathrm{L}$ of acetonitrile was added, and the mixture was centrifuged at $12,298 \times g$ for 5 minutes at $4^{\circ} \mathrm{C}$. Two microliters of the supernatant was injected into the HPLC-MS/ MS system.

\section{Statistical analyses}

The EEG FC and NP test scores were compared between baseline (before perampanel treatment) and follow-up (after 25 weeks of perampanel treatment). To determine the normality of the data, the Kruskal-Wallis test was used. Differences in the overall connectivity strength between baseline and follow-up were evaluated using the Wilcoxon signed-rank test because the data did not follow a normal distribution. Statistical analysis of electrode pairwise connectivity strength differences was performed using the nonparametric permutation test with pixel-based multiple testing correction. ${ }^{21}$

The changes in connectivity that were revealed to be significant after pixel-based correction were analyzed to determine whether they were correlated with the perampanel serum levels. Spearman's rank correlation coefficient was used because the data were not normally distributed.

A two-tailed significance level of $\mathrm{P}<0.05$ was used for all statistical analyses. Statistical analyses were performed using MATLAB 2019b.

\section{Results}

\section{Clinical information and cognitive functions}

Seventeen patients completed the present study. An error occurred in the EEG recording of an electrode (P8) in one patient, so the EEG analysis was performed in 16 patients.

The mean age of the 16 patients was 33.3 years (range, 21-65 years), and eight were female (50.0\%). The mean length of education was 14.8 years, with most $(75.0 \%)$ reporting having received college education. The average maintenance dose of perampanel was $5.75 \mathrm{mg} /$ day (range, $4-8 \mathrm{mg} /$ day). Fourteen patients were prescribed perampanel as adjunctive treatment (87.5\%), and 14 had partial epilepsy (87.5\%). Eight patients (50.0\%) showed a more than $50 \%$ reduction in seizure frequency after taking perampanel.

The RCFT delayed recall test score was significantly increased at follow-up compared with that at baseline (follow-up, 22.94; baseline, 20.03; P = 0.004). NP tests other than the RCFT delayed recall test showed no significant change following perampanel treatment. Detailed statistical information regarding the NP test scores is presented in our previous study. ${ }^{7}$ 


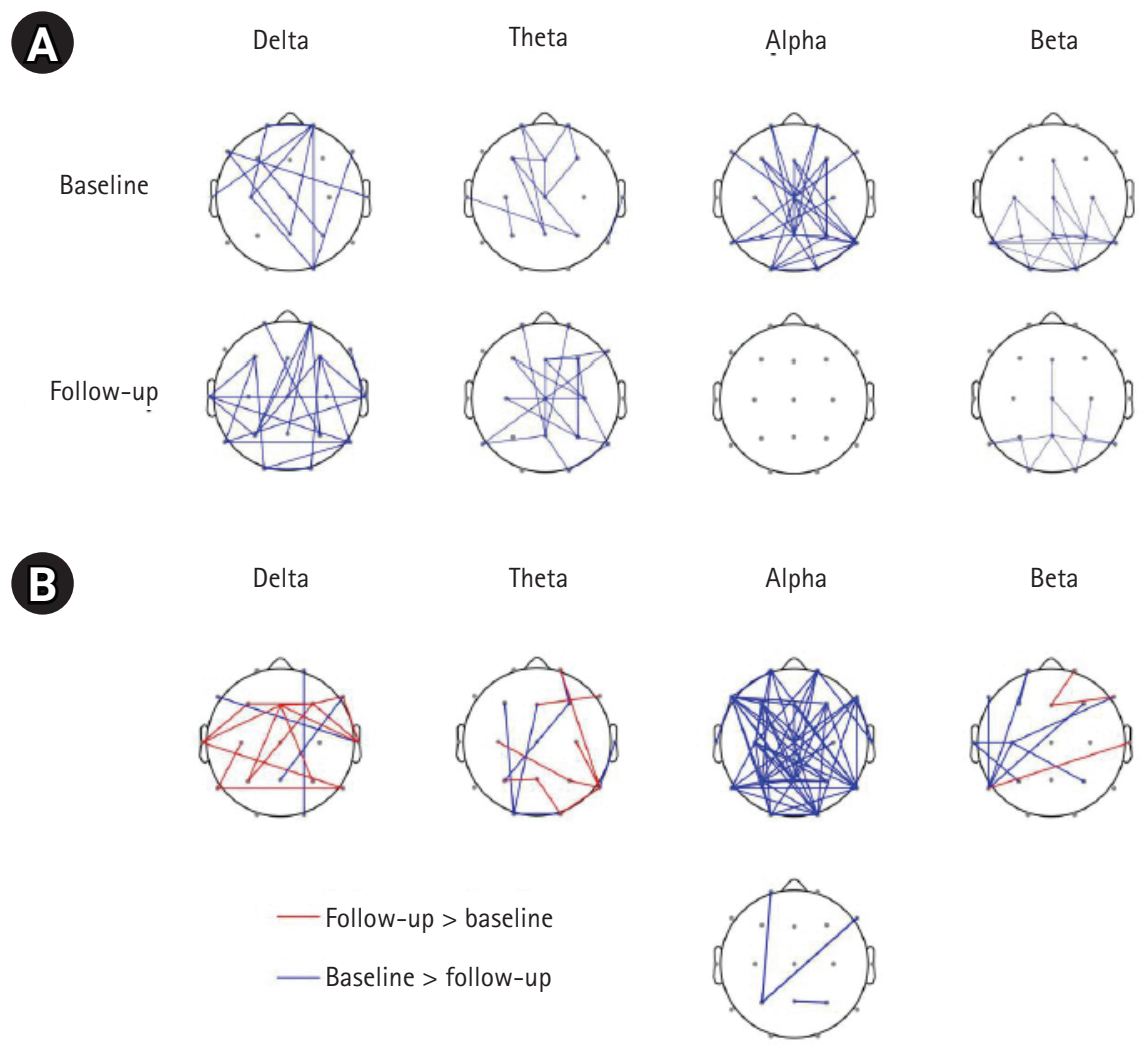

Fig. 1. (A) Overall spatial pattern of functional connectivity (FC) at baseline and follow-up. The lines indicate connections representing the highest $10 \%$ of all weighted phase lag index values at each frequency. (B) Differences in FC between baseline and follow-up. The red and blue lines indicate connections with significantly increased and decreased FC at follow-up, respectively, compared with those at baseline. The upper panel shows the significant differences in connectivity before multiple testing corrections (uncorrected, $\mathrm{P}<0.05$ ). The lower panel shows the differences in FC between baseline and follow-up after pixel-based multiple testing corrections (family-wise error rate $<0.05$ ). No significant difference was found in FC in the delta, theta, and beta bands after correction.

\section{Functional connectivity analysis}

FC differences between baseline and follow-up are summarized in Fig. 1 and Table 1.

\section{1) Global connectivity strength}

The overall connectivity strength of each frequency band was calculated by averaging the wPLI over all electrode pairs for each frequency band (Table 1). The Wilcoxon signed-rank test revealed no significant difference for all frequency bands. Although not statistically significant, a small decrease was found in the overall connectivity strength of the alpha band in the follow-up compared with that at baseline (follow-up, 0.5131; baseline, 0.5476; $\mathrm{P}=0.0980$ ).

\section{2) Overall spatial pattern of functional connectivity}

The largest $10 \%$ of the wPLI values for each frequency band were selected to observe the change in the overall spatial pattern of FC at baseline and follow-up. The overall patterns were generally similar in the delta, theta, and beta frequency bands, with an increase in the temporal and parietal regions at follow-up in the delta and theta bands and a decrease in the beta band. The alpha band FC was noticeably decreased at follow-up (Fig. 1A).

\section{3) Electrode pairwise functional connectivity comparison}

Electrode pairwise FC comparison before correction showed increases in FC in the delta band and decreases in the alpha band after perampanel treatment. After pixel-based multiple testing corrections (family-wise error rate $<0.05$ ), three electrode pairs of connections in the alpha band were significantly reduced at follow-up compared with that at baseline (Fig. 1B). The three connections were FP1-P3 ( $\mathrm{P}=0.0008)$, F8-P3 ( $\mathrm{P}=0.0110)$, and $\mathrm{P} 4-\mathrm{Pz}(\mathrm{P}=0.0208)$.

\section{Correlation of functional connectivity with perampanel serum levels}

The perampanel serum levels of 15 subjects were used for analysis because the serum level of one patient was not obtainable. The mean serum perampanel level was $238.5 \pm 150.6 \mathrm{ng} / \mathrm{mL}$ 
Table 1. Overall strength of functional connectivity at baseline and follow-up

\begin{tabular}{|c|c|c|c|}
\hline Functional connectivity $(\mathrm{Hz})$ & Baseline & Follow-up & P-value \\
\hline Delta (2-4) & $0.5877 \pm 0.0108$ & $0.5937 \pm 0.0194$ & 0.4691 \\
\hline Theta (4-8) & $0.4394 \pm 0.0219$ & $0.4401 \pm 0.0245$ & 0.9588 \\
\hline Beta $(12-30)$ & $0.2786 \pm 0.0223$ & $0.2749 \pm 0.0229$ & 0.5349 \\
\hline
\end{tabular}

Values are presented as mean \pm standard deviation.

(range, 47.9-504 ng/mL). No electrode pair was significantly correlated with the perampanel serum levels. The Spearman correlation coefficients of the FP1-P3, F8-P3, P4-Pz electrode pairs were $r=0.3036(P=0.2708), r=-0.2821(P=0.3072)$, and $r$ $=0.1893(\mathrm{P}=0.4984)$, respectively.

\section{Discussion}

The present study was the first to investigate the long-term effect of perampanel on EEG FC. Previous studies have reported that the adverse effects of perampanel on cognition are minor. ${ }^{6-9}$ Despite small or no clinical changes, other studies, including ours, showed significant changes in EEG spectral power after perampanel treatment. ${ }^{9,10}$ Only one study has investigated the effect of perampanel on $\mathrm{FC}^{22}$ Magnetoencephalography recorded before and 2 hours after perampanel intake in 20 healthy volunteers reported selective increases in amplitude-based FC in the alpha $(8-13 \mathrm{~Hz})$ and beta $(13-30 \mathrm{~Hz})$ bands. Our study results seem to indicate changes in the opposite direction from ours, where we observed a decrease in alpha band connectivity. However, in addition to using a different measure of FC from ours, this study only demonstrated acute effects in healthy young male subjects where subjects had significant somnolence and dizziness after taking a single dose of a 6-mg perampanel tablet. Nevertheless, the acute effects of perampanel on FC should also be explored in patients with epilepsy in the future.

The overall connectivity strength showed a tendency to decrease in the alpha band after perampanel treatment, although it was not statistically significant $(\mathrm{P}=0.0980)$. Decreases in the alpha band FC were also apparent in the overall spatial pattern. When the FC was compared electrode pairwise, the connectivity strength of three pairs (FP1-P3, F8-P3, P4-Pz) was significantly reduced after treatment in the alpha band after pixel-based multiple testing corrections (family-wise error rate $<0.05$ ). Overall, the present study showed a decrease in alpha band FC after perampanel treatment. A previous study conducted using resting-state EEG recordings of amnestic mild cognitive impairment patients with type 2 diabetes revealed significant decreases in the global mean PLI in the lower alpha $(8-10 \mathrm{~Hz})$, upper alpha (10-
$13 \mathrm{~Hz})$, and beta $(13-30 \mathrm{~Hz})$ bands. ${ }^{13}$ Another study reported decreased connections in the upper alpha $(10-13 \mathrm{~Hz})$ band measured by lagged phase synchronization in resting-state EEGs in patients with Alzheimer disease. ${ }^{14}$ A study using synchronization likelihood (SL) showed that patients with vascular dementia or mild Alzheimer disease had reduced frontoparietal SL. ${ }^{23}$ Considering that several studies have shown associations between cognitive deficits and decreases in alpha band FC, our study results may suggest the potential adverse effects of perampanel on cognitive function.

The differences between baseline and follow-up of the three electrode pairs exhibiting significant decreases were not correlated with the perampanel serum levels. Although the underlying reason for this deviation cannot be fully explained, several aspects should be considered. First, the total serum concentration, not the free concentration, was used in the present study. The total serum concentration is widely used and has been shown to generate acceptable outcomes in practice. However, because only the free form of the drug can pass the blood-brain barrier to exert an effect, the total serum levels can sometimes be misleading. ${ }^{24,25}$ Perampanel is highly bound to serum proteins (95\%), making the free fraction important in the drug effect. ${ }^{26,27}$ Factors not considered in the present study may have induced variations in the free fraction of perampanel between patients, resulting in discrepancies between the total and free serum levels. Additionally, the number of subjects was small, reducing the statistical power. Considering the substantial difference between individuals, a larger sample size may be required to detect the true differences in FC.

In this study, we reanalyzed the EEG recordings of a previous study to measure changes in phase-based FC induced by perampanel treatment. We observed significantly reduced FC in the alpha band at follow-up, particularly in frontoparietal connections, signifying the effect of perampanel on EEG FC and implying a potential adverse cognitive effect. However, the electrode pairs showing significant changes were not correlated with the perampanel serum levels, indicating the need for further studies to verify the effect of perampanel on alpha band FC. Further studies to locate the particular sites at which connections are reduced could 
lead to a better understanding of the meaning of the changes in FC found in the present study.

\section{Notes}

\section{Conflicts of interest}

No potential conflicts of interest relevant to this article was reported.

\section{Author contributions}

Conceptualization: Jung KY, Lee SK. Data curation: Ahn SJ, Lee SH. Formal analysis: Lee SH, Cha KS. Funding acquisition, Investigation: Jung KY. Methodology: Lee SH, Cha KS. Project administration: Jung KY. Visualization: Lee SH. Writing-Original Draft: Lee SH, Jung KY. Writing-Review \&amp; Editing: All authors.

\section{Acknowledgements}

This work was supported by an Institute for Information \& Communications Technology Promotion (IITP) grant funded by the Ministry of Science and ICT (MSIT), Republic of Korea (No. 2018-0-00861, Intelligent SW Technology Development for Medical Data Analysis).

\section{References}

1. Rogawski MA. Revisiting AMPA receptors as an antiepileptic drug target. Epilepsy Curr 2011;11:56-63.

2. Rogawski MA, Hanada T. Preclinical pharmacology of perampanel, a selective non-competitive AMPA receptor antagonist. Acta Neurol Scand Suppl 2013;(197):19-24.

3. Meador KJ, Yang H, Piña-Garza JE, Laurenza A, Kumar D, Wesnes KA. Cognitive effects of adjunctive perampanel for partial-onset seizures: a randomized trial. Epilepsia 2016;57:243251.

4. Piña-Garza JE, Lagae L, Villanueva V, et al. Long-term effects of adjunctive perampanel on cognition in adolescents with partial seizures. Epilepsy Behav 2018;83:50-58.

5. Hussein Z, Gidal B, Yang H, et al. Pharmacokinetic, pharmacodynamic and cognitive effects of adjunctive perampanel in adolescents with inadequately controlled partial seizures (P1.255). Neurology 2015;84(14 Suppl):P1.255.

6. Meschede C, Witt JA, Rademacher M, von Wrede RD, Elger $\mathrm{CE}$, Helmstaedter C. Evaluating the longer-term cognitive effects of adjunctive perampanel compared to lacosamide in a naturalistic outpatient setting. Seizure 2018;58:141-146.
7. Ahn SJ, Kim TJ, Cha KS, et al. Effects of perampanel on cognition and quantitative electroencephalography in patients with epilepsy. Epilepsy Behav 2021;115:107514.

8. Liguori C, Spanetta M, Izzi F, et al. Perampanel increases cortical EEG fast activity in child and adult patients affected by epilepsy: a quantitative EEG study. Clin EEG Neurosci 2020 Aug 7 [Epub]. https://doi.org/10.1177/1550059420947936.

9. Fries P. A mechanism for cognitive dynamics: neuronal communication through neuronal coherence. Trends Cogn Sci 2005; 9:474-480

10. Beltramini GC, Cendes F, Yasuda CL. The effects of antiepileptic drugs on cognitive functional magnetic resonance imaging. Quant Imaging Med Surg 2015;5:238-246.

11. Stam CJ, Nolte G, Daffertshofer A. Phase lag index: assessment of functional connectivity from multi channel EEG and MEG with diminished bias from common sources. Hum Brain Mapp 2007;28:1178-1193.

12. Wang YP, Gorenstein C. Assessment of depression in medical patients: a systematic review of the utility of the Beck Depression Inventory-II. Clinics (Sao Paulo) 2013;68:1274-1287.

13. Johns MW. A new method for measuring daytime sleepiness: the Epworth sleepiness scale. Sleep 1991;14:540-545.

14. Devinsky O, Ronsaville D, Cox C, Witt E, Fedio P, Theodore WH. Interictal aggression in epilepsy: the Buss-Durkee Hostility Inventory. Epilepsia 1994;35:585-590.

15. Pangman VC, Sloan J, Guse L. An examination of psychometric properties of the mini-mental state examination and the standardized mini-mental state examination: implications for clinical practice. Appl Nurs Res 2000;13:209-213.

16. Kim H, Na DL. Normative data on the Korean version of the Boston Naming Test. J Clin Exp Neuropsychol 1999;21:127133.

17. Fastenau PS, Denburg NL, Hufford BJ. Adult norms for the Rey-Osterrieth Complex Figure Test and for supplemental recognition and matching trials from the Extended Complex Figure Test. Clin Neuropsychol 1999;13:30-47.

18. Kim JK, Kang Y. Normative study of the Korean-California Verbal Learning Test (K-CVLT). Clin Neuropsychol 1999;13:365369.

19. Sumerall SW, Timmons PL, James AL, Ewing MJ, Oehlert ME. Expanded norms for the Controlled Oral Word Association Test.J Clin Psychol 1997;53:517-521.

20. Reitan RM. The relation of the trail making test to organic brain damage. J Consult Psychol 1955;19:393-394.

21. Nichols TE, Holmes AP. Nonparametric permutation tests for functional neuroimaging: a primer with examples. Hum Brain Mapp 2002;15:1-25. 
22. Routley BC, Singh KD, Hamandi K, Muthukumaraswamy SD. The effects of AMPA receptor blockade on resting magnetoencephalography recordings. J Psychopharmacol 2017;31:15271536.

23. Babiloni C, Ferri R, Binetti G, et al. Fronto-parietal coupling of brain rhythms in mild cognitive impairment: a multicentric EEG study. Brain Res Bull 2006;69:63-73.

24. Beran RG, Lewis JH, Nolte JL, Westwood AP. Use of total and free anticonvulsant serum levels in clinical practice. Clin Exp Neurol 1985;21:69-77.
25. Chan K, Beran RG. Value of therapeutic drug level monitoring and unbound (free) levels. Seizure 2008; 17:572-575.

26. Patsalos PN, Zugman M, Lake C, James A, Ratnaraj N, Sander JW. Serum protein binding of 25 antiepileptic drugs in a routine clinical setting: a comparison of free non-protein-bound concentrations. Epilepsia 2017;58:1234-1243.

27. Franco V, Crema F, Iudice A, Zaccara G, Grillo E. Novel treatment options for epilepsy: focus on perampanel. Pharmacol Res 2013;70:35-40. 